\title{
HOW THE EVOLUTION OF TEACHING MAY HELP UNDERSTANDING THE EVOLUTION OF LANGUAGE
}

\author{
PETER GÄRDENFORS ${ }^{* 1,2}$ \\ *Corresponding Author: Peter.Gardenfors@lucs.lu.se \\ ${ }^{1}$ Department of Philosophy, Lund University, Lund, Sweden \\ ${ }^{2}$ Stellenbosch Institute for Advanced Studies, Wallenberg Research Centre at Stellenbosch \\ University, Marais Street, Stellenbosch 7600, South Africa
}

\section{Introduction}

Teaching is present in all extant human societies. On the other hand, intentional teaching within other species is very limited. In an analysis of the evolution of teaching, demonstration and pantomime come out as pivotal capacities. The aim of this paper is to spell out the relevance of this for the evolution of language.

\section{The Evolution of Teaching}

Gärdenfors and Högberg (2017) distinguish six levels of intentional teaching: (1) intentional approval/disapproval, (2) drawing attention, (3) demonstrating and pantomime, (4) communicating concepts, (5) explaining concept relations and (6) narrating. They hypothesize that level after level has been added during the evolution of teaching. They analyse communicative requirements for the levels, concluding that displaced communication is required for level 4 and symbolic language only for levels 5-6.

\section{The Central Role of Demonstration and Pantomime}

It is only humans who teach according to levels (3)-(6). A central question is therefore why only hominins have the capacity to demonstrate and pantomime, that is, to reach level (3). This capacity seems to have generated a breakthrough in hominin teaching and in transmission of culture. When a teacher demonstrates to a learner how to perform a certain task, the demonstrator actually performs the actions involved in the task, commonly using material culture. In pantomime the mimer (teacher) only performs the movements of the actions in the task. I argue that the capacity to pantomime has been pivotal in the evolution of communication.

For pantomime (but not for demonstration), the addressee must understand that the teacher intends the pantomime to stand for a real action and that the teacher intends the addressee to realize this. Zlatev et al. (2005) call this the 
'communicative sign function'. Another important difference with respect to demonstration is that pantomime is displaced (Zywiczynski et al. 2016, sect. 3.8). However, pantomime is neither conventional nor symbolic.

\section{From Pantomime to Language}

Following Gärdenfors and Högberg (2017), I instead view the evolution of teaching as a series of stages that I argue can be matched to different stages in the evolution of communication. There are two main functions for pantomime: The primary is an invitation to copy - the teaching function. The secondary is a communicative function. This distinction also shows up when determining what is the intention of a pantomime. A teacher can pantomime an action that the teacher wants the student to copy, and an action can be pantomimed as part of a message (request, command, warning, narrative, etc.). In the gesture literature, the communicative use of pantomime has been in focus.

Pantomime has been argued to be a precursor to protosign and protolanguage. Arbib (2012) suggests that protosign develops by conventionalization out of pantomime and other gestures. A crucial feature is that pantomime provides an open set of gestures that can be generated to create new meanings. It should be noted that conventions presume common knowledge among the signers), something that requires higher order mindreading. Arbib (2012, p. 231) then proposes that protospeech builds on protosign in an 'expanding spiral'.

Gärdenfors (2013) argues that symbolic language is necessary for the advanced forms of cooperation that have evolved along the hominin line. Teaching should, however, also be seen as a form of cooperation and the later stages in the model of Gärdenfors and Högberg (2017) require advanced forms of communication. I conclude that pantomime is necessary for the emergence of any of these forms.

\section{References}

Arbib, M. (2012). How the Brain Got Language: The Mirror System Hypothesis. Oxford: Oxford University Press.

Gärdenfors, P. (2013). The evolution of semantics: Sharing conceptual domains. In The Evolutionary Emergence of Language, eds. Botha, R. and Everaert, M. (Oxford: Oxford University Press), 139-159.

Gärdenfors, P. \& Högberg, A. (2017). The archaeology of teaching and the evolution of Homo docens. Current Anthropology, 58, 188-201.

Zlatev, J., Persson, T. \& Gärdenfors, P. (2005a). Bodily mimesis as 'the missing link' in human cognitive evolution. Lund: Lund University Cognitive Studies 121.

Zywiczynski, P., Wacewicz, S. \& Sibierska, M. (2016). Pantomime for language evolution research. Topoi, DOI 10.1007/s11245-016-9425-9. 